\section{Bivar: A program for generating correlated random numbers}

\author{
JEFF MILLER \\ University of Otago, Dunedin, New Zealand
}

This article describes Bivar, a program that generates random numbers from a wide variety of bivariate distributions. Numbers are written to an ASCII output file, from which they can be read as needed by programs simulating stochastic processes. The program runs under the DOS operating system on IBM-PC compatibles with a 386 or later processor, and it is free for educational and noncommercial use.

Many stochastic models in the behavioral sciences characterize an individual's actions in terms of a set of independent random variables (see, e.g., Luce, 1986; Massaro \& Friedman, 1990). For example, the response time (RT) on an individual trial might be modeled in terms of a race between two independent response processes active on that trial (as in Raab, 1962), or an "old versus new" judgment might be modeled in terms of independent familiarity and recognition processes (as in Yonelinas \& Jacoby, 1994). Nonetheless, it is easy to imagine various sources of correlation between random variables characterizing an individual's performance. For example, individuals change continuously, owing to fluctuations in arousal, fatigue, attention, and so forth. It is easy to imagine that such changes would have effects on more than one of the random variables used to characterize the individual, thereby producing some correlation across trials in the values of these variables. Thus, it seems prudent to study the effects of moderate correlations between random variables on the predictions of our models, to make sure that violations of the independence assumption would not drastically change their predictions regarding summary statistics (e.g., mean RT). Since many such models become mathematically intractable if dependence is allowed (as in, e.g., Colonius, 1987), such studies are at present best conducted by computer simulation.

This article describes a computer program that can be used to generate correlated random variables from a wide variety of probability distributions for use in such simulations. The program, called Bivar, runs on IBM-PC compatible microcomputers and is freely available for academic use. The program was designed for batch use to

\footnotetext{
I thank Ellen Hertz of the National Highway Traffic Safety Administration, United States Department of Transportation, for suggesting the mixture structure, and Andrew Heathcote and two anonymous reviewers for helpful suggestions concerning this manuscript as well as the program and its documentation. Correspondence concerning this article should be addressed to J. Miller, Department of Psychology, University of Otago, Dunedin, New Zealand (e-mail: miller@otago. ac.nz).
}

facilitate simulation runs without operator intervention, so program parameters are specified with an ASCIl file prepared in advance. The generated random numbers are written to an ASCII file, from which they can be read and processed by a simulation program or statistical package.

In its primary mode of operation, Bivar generates $(X, Y)$ pairs of random variables from a bivariate probability distribution having preselected marginal distributions and correlation. The marginal distributions, whose cumulative forms will be denoted $F_{x}(X)$ and $F_{y}(Y)$, can be selected from a wide variety of distributional families and need not be the same. For example, the marginal distribution of $X$ could be a normal distribution with mean 0 and variance 1 , and that of $Y$ could be an exponential with rate parameter equal to one. The basic distributional families that have been implemented are listed in Table 1; in addition, the program can use other marginal distributions derived from these by truncation, mixturing, convolution, and computation of order statistics, as in the program Cupid (Miller, 1998).

The program provides three different structures for implementing a bivariate correlation while maintaining the exact desired marginals. With some models, the choice among structures may be somewhat arbitrary, because the modeler will have no idea which of these structures is most similar to the true one. Nonetheless, the three structures are quite different from one another, so by examining the effects of all three types of correlations, the modeler can gain some insight into the robustness of the model's predictions in the presence of different types of violations of the independence assumption. Unfortunately, these structures do not generalize easily to multivariate problems, as does an approximate-marginal method suggested by Bradley and Fleisher (1994).

One bivariate structure is a transformation of the bivariate normal distribution (cf. Bradley, 1993). This structure produces bivariate random numbers more or less resembling a bivariate normal distribution, like those illustrated in the first panel of Figure 1. With this structure, each $(X, Y)$ pair is generated as follows: First, using a standard technique (e.g., that of Graybill, 1969), a pair $\left(Z_{x}, Z_{y}\right)$ is generated randomly from a bivariate normal distribution with marginal means of zero, marginal standard deviations of one, and a prespecified correlation $\rho$. Second, the pair $\left(P_{x}, P_{y}\right)$ is computed, where $P_{x}$ and $P_{y}$ are the cumulative probabilities within the standard normal distribution associated with $Z_{x}$ and $Z_{y}$, respectively. Third, $X$ is taken as $F_{x}^{-1}\left(P_{x}\right)$, and $Y$ is taken as $F_{y}^{-1}\left(P_{y}\right)$. It can be seen that this method does indeed produce the desired marginals for $X$ and $Y ; P_{x}$ and $P_{y}$ are both uniformly distributed between zero and one by construction, so the different values of $X$ and $Y$ occur with the desired marginal probabilities. Moreover, the correlation of $X$ and $Y$ will have the same sign as $\rho$, although it will not necessarily have the same magnitude (cf. Bradley, 1993; 
Table 1

List of Available Marginal Probability Distributions

\begin{tabular}{ll} 
Beta & Logistic \\
Binomial & Log-normal \\
Cauchy & Normal \\
Chi-square & Pearson's $r$ \\
Ex-Gaussian & Poisson \\
Exponential & Rayleigh \\
Extreme value & Studentized range \\
$F$ (Fisher's) & Student's $t$ \\
Gamma & Triangular \\
Laplace & Uniform \\
Lilliefors & Weibull \\
$A^{\prime}$ and $d^{\prime}$ (from signal detection theory) \\
\hline
\end{tabular}

Bradley \& Fleisher, 1994). As described later, the value of $\rho$ can be adjusted to obtain the desired numerical value for the correlation between $X$ and $Y$.

A second bivariate structure uses a mixture of maximally correlated pairs and uncorrelated pairs. With this structure, $X$ is randomly generated from its marginal distribution. Then, with a preselected probability $p, Y$ is taken as $F_{y}^{-1}\left(F_{x}(X)\right)$ (or, if a negative correlation is desired, as $\left.F_{y}^{-1}\left(1-F_{x}(X)\right)\right)$. With probability $1-p$, however, $Y$ is chosen from its marginal distribution independently of $X$. A set of random numbers produced using this method is shown in the second panel of Figure 1; the dense set of points on a $45^{\circ}$ line within the scattergram represent the perfectly correlated observations, and the remaining scattered uncorrelated points represent the independent ones. Again, the method produces the correct marginals. $X$ is chosen directly from its desired marginal distribution, and $Y$ is simply a mixture of two different cases, each of which has the desired marginal distribution for $Y$. The strength of the correlation between $X$ and $Y$ is controlled by the value of the mixture probability $p$, which may be adjusted to obtain the desired correlation as described later.

The third bivariate structure divides each marginal probability distribution into percentile regions or "bands." With four bands, for example, each distribution is divided into bands of $0 \%-25 \%, 25 \%-50 \%, 50 \%-75 \%$, and $75 \%-$ $100 \%, X$ is generated randomly from its marginal distribution, and the program determines which band $X$ came from. Then $Y$ is generated randomly from the same band if a positive correlation is desired, and from the complementary band for a negative correlation (i.e., if one band goes from $a \%$ to $b \%$, the complementary band goes from $(100-b)$ to $(100-a) \%)$. The third panel of Figure 1 illustrates a set of points generated with this method. Approximately two thirds of the points were generated with two bands and thus lie within the lower left and upper right quadrants of the scattergram. The remaining third of the points were generated with three bands, and thus also define - albeit less clearly - a region with both variables in the middle third of their marginals. This structure also yields the desired marginals. $X$ is generated directly from its marginal, and $Y$ is generated from one of a set of equally likely bands within its marginal. The strength of the correlation between $X$ and $Y$ increases with the number of bands, which can be adjusted to give the desired correlation as described later.

A limitation of the band structure is that with an integral number of bands it may not be possible to produce a desired correlation. With normal and exponential marginals, for example, the correlation is .555 with two bands and .697 with three bands, so an intermediate correlation could not be produced with an integral number of bands. To overcome this limitation, the band technique was generalized to use a mixture of different numbers of bands to generate different $(X, Y)$ pairs. For example, if two
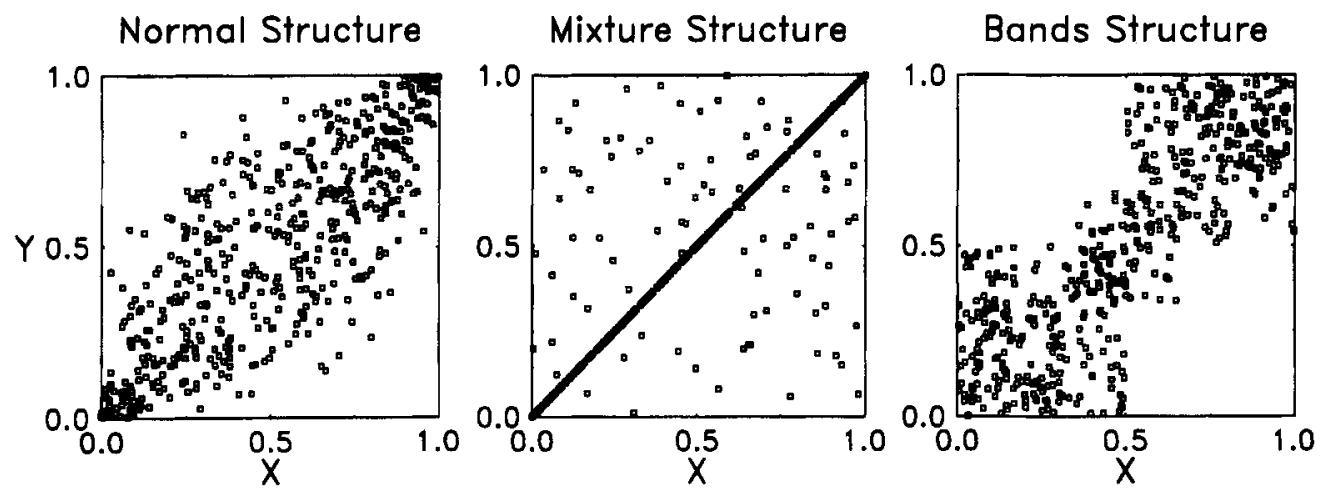

Figure 1. Scatterplots of three data sets generated by Bivar. Each panel was generated by using a different bivariate structure, as indicated at the top. For each panel, the underlying marginal distributions of $X$ and $Y$ were uniform distributions ranging from zero to one, the undertying bivariate distribution had a true correlation of .80 , and 500 $X, Y$ pairs were generated. The observed correlations for the three generated data sets ranged from .79 to .81 . For the normal structure, the undertying correlation of the bivariate normal distribution was .813 ; for the mixture structure, the probability of a perfectly correlated pair was .80; for the bands structure, the number of bands was $\mathbf{2 . 3 6 5}$, which means that observations were generated from two underlying bands with probability .635 and from three undertying bands with probability .365 . 
bands are used with probability .6 and three bands are used with probability .4, the overall correlation is .612 . In fact, any intermediate correlation can be produced by adjusting this probability. By convention, then, the program allows the number of bands to be a decimal number rather than an integer, and it uses the fractional part to determine the probabilities of the smaller versus larger number of bands.

As already noted, each of the three different bivariate structures employs a single parameter to control the magnitude and sign of the correlation between $X$ and $Y$. For the bivariate normal structure, this is the $\rho$ of the bivariate normal. For the mixture structure, it is the probability $p$ of a maximally correlated pair. For the bands structure, it is the number of bands. In practice, however, the researcher will usually want to specify the bivariate structure in terms of the numerical value of the correlation between $X$ and $Y$ rather than in terms of one of these controlling parameters. To facilitate this process, the program has a search mode in which the user specifies the marginal distribution, the bivariate structure, and the desired correlation. The program then uses a numerical search algorithm to locate the appropriate value of the controlling parameter to obtain the desired correlation (cf. Bradley \& Fleisher, 1994). During the search process, the program does not actually generate random data points, but instead computes the actual correlation produced by each candidate value of the controlling parameter with an $N \times N$ grid approximation of the bivariate distribution, where $N$ is specified by the user. The value of the controlling parameter is adjusted iteratively until the desired correlation is reached.
The program output includes one file containing the generated data points, and another file summarizing the data set. The latter file includes the $X, Y$ correlation within the generated data set, the univariate means and standard deviations of $X$ and $Y$, and the value of a chi-square statistic used to test whether each univariate data set deviates significantly from the intended underlying distribution.

Table 2 summarizes the results of 45 sample runs, defined by 15 different pairs of marginal distributions, each of which was used with all three possible bivariate structures. For all runs, Bivar was used to generate data sets of 10,000 pairs from an underlying bivariate distribution with the controlling parameter adjusted to produce a correlation of .60. The obtained sample correlations, shown in the $r$ columns, are all quite close to this target value. In addition, the fits of the generated $X \mathrm{~s}$ and $Y$ s to the desired marginal distributions were evaluated with a $\chi^{2}$ test for each random variable. Of the $90 \chi^{2}$ tests thus carried out, 83 yielded nonsignificant results, indicating that the deviations from the expected marginals were within chance limits. The remaining seven tests were significant at $p<.10$ or less, but a few significant deviations are expected by chance with repeated hypothesis testing of this sort. Consistent with the chance interpretation of these significant deviations, 10 further repetitions of each of the seven significant results (i.e., 70 further tests in all) yielded a total of seven additional results significant at the level of $p<.1$, exactly the expected number by chance.

In addition to the program file Bivar.exe, Bivar comes with a postscript file containing a printable manual and an ASCII version of the manual that can be inspected on

Table 2

Results Obtained in 45 Test Runs Using Each of 15 Different Pairs of Marginal Distributions With Each of the Three Available Bivariate Structures

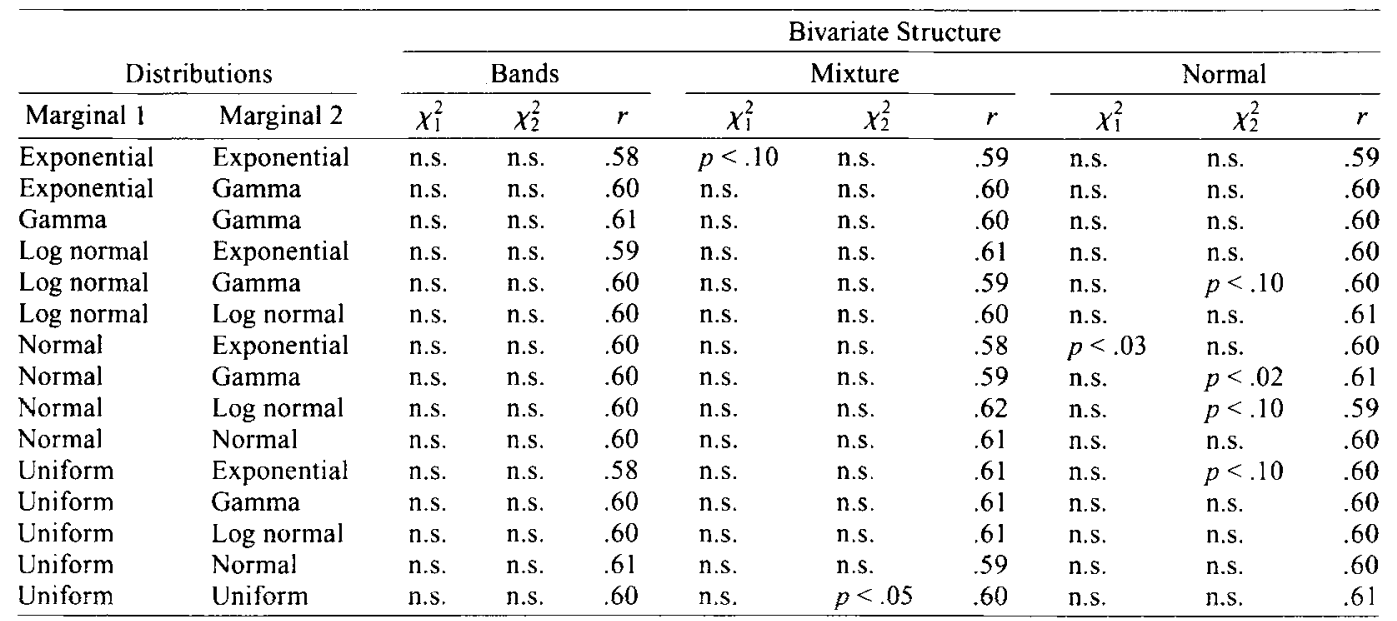

Note-For each test run, 10,000 pairs of observations were generated with a target correlation of 60 . Each test run is summarized in terms of the observed sample correlation $(r)$ and the attained significance level of $\chi^{2}$ tests evaluating the fit of the generated observations to the desired marginal distributions (n.s. indicates $p>10$ ). The underlying normal, log normal, and uniform marginals were those with $\mu$ and $\sigma$ equal to 100 and 15 , respectively. The underlying gamma marginal had a shape parameter set arbitrarily to five and rate chosen to produce $\sigma=15$, and the underlying exponential also had a rate chosen to produce $\sigma=15$. 
line. The complete version is available by sending a formatted 3.5-in. high-density floppy disk and a stamped, self-addressed mailer to the author, and it is also available by ftp from common archive sites in the file Bivar 1_1.Zip (the numbers in the file name may change as future versions are developed). The author may be contacted for further information about obtaining the program electronically.

\section{REFERENCES}

BRADLEY, D. R. (1993). Multivariate simulation with DATASIM: The Mihal and Barrett study. Behavior Research Methods, Instruments, \& Computers, 25, 148-163.

BRADLEY, D. R., \& FLEISHER, C. L. (1994). Generating multivariate data from nonnormal distributions: Mihal and Barrett revisited. Behavior Research Methods, Instruments, \& Computers, 26, 156-166.

Colonius, H. (1987). Modeling dependent processing in reaction time analysis. In E. E. Roskam \& R. Suck (Eds.), Progress in mathematical psychology (Vol. 1, pp. 197-207). Amsterdam: Elsevier.

GraybilL, F. A. (1969). Introduction to matrices with applications in statistics. Belmont, CA: Wadsworth.

LuCE, R. D. (1986). Response times: Their role in inferring elementary mental organization. Oxford: Oxford University Press.

Massaro, D. W., \& Friedman, D. (1990). Models of integration given multiple sources of information. Psychological Review, 97, 225252.

MilleR, J. [O.] (1998). Cupid: A program for computations with probability distributions. Behavior Research Methods, Instruments, \& Computers, 30, 544-545

RAAB, D. H. (1962). Statistical facilitation of simple reaction times. Transactions of the New York Academy of Sciences, 24, 574-590.

Yonelinas, A. P., \& JACOBY, L. L. (1994). Dissociations of processes in recognition memory: Effects of interference and of response speed. Canadian Journal of Psychology, 48, 516-534.

(Manuscript received April 25, 1997; revision accepted for publication August 21, 1997.) 